\title{
Using the ESS Maximum Principle to Explore Root-shoot Allocation, Competition and Coexistence
}

\author{
TANia L. S. Vincent† AND Thomas L. Vincent \\ Department of Ecology, Evolution and Behavior, 100 Ecology Building, 1987 Upper Buford \\ Circle, St Paul, MN 55108, and the Department of Aerospace and Mechanical Engineering, \\ University of Arizona, Tucson, Arizona 85721, U.S.A.
}

(Received on 31 January 1995, Accepted in revised form on 30 November 1995)

\begin{abstract}
Competition between plants that differ in root-shoot allocation has been modelled using consumer-resource equations where competition occurs only through the utilization of resources. Provided that such models can be put into an evolutionary game setting, we show that conditions for coexistence can be analysed using the ESS maximum principle from evolutionary game theory. We demonstrate this approach using a modified version of a consumer-resource model from Reynolds \& Pacala (1993). Allocation of biomass by the consumers to root or shoot is expressed as an evolutionary strategy. Since stability conditions are defined by the ESS maximum principle, aspects of coexistence can be determined without analyses of Jacobian matrices. Using the ESS maximum principle, we confirm that coexistence between two or more species is not possible with this model. We show that this result depends on the consumer growth rate being a linear function of allocation strategy and does not depend on the form of the (nonlinear) nutrient and light availability equations. In addition, we show that species coexistence is possible if the model is modified to include a nonlinear dependence of consumer growth rate on allocation strategy. We give an example where coexistence between two species is obtained.
\end{abstract}

(C) 1996 Academic Press Limited

\section{Introduction}

Since allocation strategies are known to affect plant growth under different resource conditions (Grime, 1977; Chapin, 1980; Bloom et al., 1985; Tilman, 1988), it follows that models of plant resource consumption and growth should include these strategies. Such models should also include the effects of competition via resource consumption between plants with different allocation strategies. For example, Tilman's (1988) model ALLOCATE, a computer simulation based on his model of plant and resource dynamics (Tilman, 1980, 1982), was developed to simulate plant competition for light and soil resources. He assumed that each plant species

$\uparrow$ Author to whom correspondence should be addressed at: PWS Science Center, PO Box 705, Cordova, AK 99574, U.S.A.

E-mail: tania@cdr.lter.umn.edu. influences its own growth and the growth of others only through the consumption of essential light and soil resources, and that plant species only differ in how they allocate biomass between roots, stems, leaves, and seeds. Reynolds \& Pacala (1993) simplified this model by restricting biomass allocation to roots and leaves which allowed them to look at some aspects of plant competition both analytically and via simulation. Both of these models have generated a number of useful insights. Succession from a single initial dominant species through a series of other dominants ending with a final dominant species was obtained. In addition, for both models, stable coexistence between species using different allocation strategies was not possible. Reynolds \& Pacala (1993) were able to determine initial and final dominant strategies and directions of succession as well as situations where succession was arrested before reaching the final dominant. 
Here we use a different approach to examine the model presented by Reynolds \& Pacala (1993; hereafter designated R \& P). We formulate their equations as an evolutionary game, with biomass allocation as evolutionary strategies. Using the ESS maximum principle (Brown \& Vincent, 1987; Vincent \& Brown, 1988; Vincent et al., 1995), we duplicate the analytical results of $\mathrm{R} \& \mathrm{P}$ (that only one species can exist at equilibrium) and demonstrate that similar computer simulations can be obtained without lengthy simulation runs that use the dynamics of a large number of species at the same time (e.g. 99 species in $\mathrm{R} \& \mathrm{P}$ ). In addition, we show that arrested succession does not occur under an evolutionary scenario and also how stability conditions differ between the approach used with the ESS maximum principle and the approach used by both Tilman (1980) and R \& P. We also show that by incorporating nonlinear consumer growth into the model, coexistence between two or more species becomes possible.

\section{The ESS Maximum Principle}

There are several key concepts and definitions involved in order to use the ESS maximum principle (Brown \& Vincent, 1987; Vincent \& Brown, 1988, 1989; Vincent et al., 1995). Since the terminology may not be familiar, we will elucidate these concepts and definitions by employing a tutorial model. This model is for illustration only, and is not intended to be representative of any particular biological system. The theory in no way depends on it.

Imagine a community composed of four interacting populations whose densities are represented by $x_{1}, x_{2}, z_{1}$, and $z_{2}$. The population dynamics of this community are modelled by multiplying the fitness function for individuals within each population with the density of that population. Within each population, we consider all individuals to be identical. Thus, the fitness function for each individual within a population is the same as the fitness function for the population itself. The fitness function for each population is made up of two components: the population densities $x_{1}, x_{2}, z_{1}, z_{2}$, as well as parameters that determine the impact of population interactions. If these parameters can evolve, then they are called strategies. Since the individuals within a population are identical, they all have the same strategy. Thus, we consider our community to be composed of populations of strategies. Let us assume that a strategy $u_{1}$ has population density $x_{1}$, a strategy $u_{2}$ has population density $x_{2}$, a strategy $v_{1}$ has $z_{1}$, and a strategy $v_{2}$ has $z_{2}$. These strategies have the potential to evolve to any value allowed under genetic constraints. For example, the $u$ and $v$ strategies are only allowed to take on values defined by

$$
\begin{aligned}
& u_{\text {min }} \leqslant u_{i} \leqslant u_{\text {max }} \\
& v_{\text {min }} \leqslant v_{i} \leqslant v_{\text {max }}
\end{aligned}
$$

where $i=1,2$.

Two or more populations are considered to be evolutionarily identical if the individuals of these populations have available the same set of strategy choices and if the ecological consequences of using a given strategy are the same. Populations $x_{1}$ and $x_{2}$ both have the same set of strategy choices. However, we have not specified fitness functions for our populations so we cannot determine whether these populations are evolutionarily identical yet. Let the dynamics for each population in our community be given by

$$
\begin{aligned}
& \dot{x}_{1}=\left[u_{1}-\left(u_{1} x_{1}+u_{2} x_{2}\right)-\left(v_{1} z_{1}+v_{2} z_{2}\right)\right] x_{1} \\
& \dot{x}_{2}=\left[u_{2}-\left(u_{1} x_{1}+u_{2} x_{2}\right)-\left(v_{1} z_{1}+v_{2} z_{2}\right)\right] x_{2} \\
& \dot{z}_{1}=\left[v_{1}\left(x_{1}+x_{2}\right)-\left(z_{1}+z_{2}\right)\right] z_{1} \\
& \dot{z}_{2}=\left[v_{2}\left(x_{1}+x_{2}\right)-\left(z_{1}+z_{2}\right)\right] z_{2}
\end{aligned}
$$

where the fitness function for each population is given by the term in brackets. Note that the fitness functions for populations $x_{1}$ and $x_{2}$ have identical form. Since $u_{1}$ and $u_{2}$ satisfy the same constraints, it follows that populations $x_{1}$ and $x_{2}$ are evolutionarily identical. Likewise for $z_{1}$ and $z_{2}$.

A convenient way to specify the fitness function for every evolutionarily identical group of populations is to use a fitness generating function (G-function). The G-function for a group of evolutionarily identical populations (e.g. $x_{1}, x_{2}$ ) is a function which contains not only all of the variables in each population's fitness function but, in addition, has a "dummy strategy" variable with the property that the fitness function, for any population, can be obtained by replacing the "dummy strategy" variable with the particular population's strategy. The fitness generating functions (G-functions) for each evolutionarily identical group are given by

$$
\begin{aligned}
G_{x} & =u-\left(u_{1} x_{1}+u_{2} x_{2}\right)-\left(v_{1} z_{1}+v_{2} z_{2}\right) \\
G_{z} & =v\left(x_{1}+x_{2}\right)-\left(z_{1}+z_{2}\right)
\end{aligned}
$$

where $u$ and $v$ represent the dummy strategies. It follows that the fitness function for the population $x_{1}$ can be obtained by replacing $u$ with $u_{1}$ in $G_{x}$ etc. 
A model of a community, such as our tutorial system, may come to an ecological equilibrium ( $\dot{x}_{1}=\dot{x}_{2}=\dot{z}_{1}=\dot{z}_{2}=0$ ) under many different strategy sets $\left(u_{1}, u_{2}, v_{1}, v_{2}\right)$. For a given set of strategies, the corresponding population densities at equilibrium $\left(x_{1}^{*} \geqslant 0, x_{2}^{*} \geqslant 0, z_{1}^{*} \geqslant 0, z_{2}^{*} \geqslant 0\right)$ will, in general, be different. The sub-set of strategies with population densities greater than zero make up a coalition vector. For example, the strategy vector $\left(u_{1}=2, u_{2}=1\right.$, $\left.v_{1}=2, v_{2}=1\right)$ will produce the ecological equilibrium $\left(x_{1}^{*}=1 / 3, x_{2}^{*}=0, z_{1}^{*}=2 / 3, z_{2}^{*}=0\right)$ in the tutorial system so that $\left(u_{1}=2, v_{1}=2\right)$ is a coalition vector for that system. Thus, at equilibrium, this system has a set of two coexisting strategies.

Our objective is to find a coalition vector which is evolutionarily stable. That is, one which will result in the same stable equilibrium densities no matter which strategies are used for the other components in the strategy vector. For example, if $u_{\min }=v_{\min }=0$ and $u_{\max }=v_{\max }=2$ for our tutorial system, then $\left(u_{1}=2\right.$, $\left.v_{1}=2\right)$ is evolutionarily stable since any strategy vector $\left(u_{1}=2,0 \leqslant u_{2} \leqslant 2, v_{1}=2,0 \leqslant v_{2} \leqslant 2\right)$ always results in the same ecological equilibrium $\left(x_{1}^{*}=1 / 3\right.$, $\left.x_{2}^{*}=0, z_{1}^{*}=2 / 3, z_{2}^{*}=0\right)$. A coalition vector with these properties is called an evolutionarily stable strategy (ESS). The concept of an ESS was first stated, in somewhat different terms, by Maynard Smith (1982).

The ESS maximum principle states that if a coalition vector is an ESS, then the G-function for each evolutionarily identical group, evaluated at equilibrium, must take on a zero maximum with respect to the "dummy strategy" for each strategy in the coalition vector (Brown \& Vincent, 1986, 1992). For the tutorial system, the ESS is obtained from

$$
\begin{aligned}
& G_{x}^{*}=u-\left(u_{1} x_{1}^{*}+u_{2} x_{2}^{*}\right)-\left(v_{1} z_{1}^{*}+v_{2} z_{2}^{*}\right) \\
& G_{\mathrm{z}}^{*}=v\left(x_{1}^{*}+x_{2}^{*}\right)-\left(z_{1}^{*}+z_{2}^{*}\right) .
\end{aligned}
$$

Considering the bounds on $u$ and $v$, it follows that $G_{x}^{*}$ takes on a maximum with respect to $u$ at $u=2$ and $G_{z}^{*}$ takes on a maximum with respect to $v$ at $v=2$. Furthermore $G_{x}^{*}=G_{z}^{*}=0$ when $\left(u_{1}=2, v_{1}=2\right)$ and $\left(x_{1}^{*}=1 / 3, x_{2}^{*}=0, z_{1}^{*}=2 / 3, z_{2}^{*}=0\right)$. Hence, $\left(u_{1}=2\right.$, $\left.v_{1}=2\right)$ satisfies the maximum principle and is, in fact, an ESS.

The ESS maximum principle covers a larger class of problems than implied by the tutorial model given above. In particular, the strategies may be vector valued, there may be any number of populations defined by a given G-function, and there may be any number of G-functions.

\section{A G-function for R \& P's Model}

In the model used by $\mathrm{R} \& \mathrm{P}$, the growth rate of a plant population is given by

$$
\begin{array}{r}
\frac{\mathrm{d} B_{p}}{\mathrm{~d} t} \frac{1}{B_{p}}=\operatorname{MIN}\left[\begin{array}{r}
\frac{r N u_{p}}{N+k_{N}}-R \\
\frac{r L\left(1-u_{p}\right)}{L+k_{L}}-R
\end{array}\right] \\
-d \quad p=1 \ldots n
\end{array}
$$

where

$$
\begin{aligned}
B_{p}= & \text { biomass of population } \mathrm{p} \\
N= & \text { available soil nutrient } \\
L= & \text { light availability } \\
u_{p}= & \text { fraction of biomass allocated to root by } \\
& \text { population } \mathrm{p} \\
r= & \text { per capita maximal rate of plant growth } \\
k_{N}= & 1 / 2 \text { saturation constant for nutrient } \\
k_{L}= & 1 / 2 \text { saturation constant for light } \\
R & =\text { density independent per capita respiration rate } \\
d & =\text { density independent per capita loss rate. }
\end{aligned}
$$

The notation used in eqn (1) is the same as $\mathrm{R} \& \mathrm{P}$ except we have used $u$ for root biomass fraction instead of $A$ in order to be consistent with the notation we are using to identify strategies. $\mathrm{R} \& \mathrm{P}$ define each population $p$ to be a species. Each species $p$ is identified by its allocation fraction $u_{p}$ where this parameter may be chosen over the fixed interval $0 \leqslant u_{p} \leqslant 1$ and we hold this same convention. In this and R \& P's model, $N$ and $L$ are not constants, but rather, are a function of resource supply and consumption. These dynamics should be kept in mind during the discussion below and will be discussed explicitly later.

Notice that each element of the growth term in eqn (1) is a linear function of strategy. For a given value of $u_{p}$ (root allocation fraction), $N$ (nutrient level), and $L$ (light level), the MIN function will choose the "nutrient-limited growth curve" (which starts at the origin) for values of $u_{p}$ between

$$
0 \leqslant u_{p} \leqslant u_{m}
$$

and it will choose the "light-limited growth curve" (which is zero at $u_{p}=1$ ) for values of $u_{p}$ between

$$
u_{m} \leqslant u_{p} \leqslant 1 .
$$

Figure 1 shows an example of these nutrient- and light-limited growth curves. The strategy $u_{m}$ at the point of intersection of the nutrient- and light-limited growth curves is given by

$$
u_{m}=\frac{L\left(N+k_{N}\right)}{2 L N+N k_{L}+L k_{N}} .
$$


Equation (4) is obtained by setting the nutrient-limited and light-limited growth curves equal to one another. The quantity $u_{m}$ maximizes the growth rate for any given level of soil nutrient and light since both resources are equally limiting (Tilman, 1980, 1982; $\mathrm{R} \& \mathrm{P}$ ). Equation (4) was used by $\mathrm{R} \& \mathrm{P}$ to obtain dominant strategies for initial conditions.

In order to put eqn (1) into a form in which we can use the ESS maximum principle, we must express the dynamics in terms of two separate differential equations. Let us now subdivide the $n$ species into two groups: nutrient-limited types, 1, and light-limited types, 2. Nutrient-limited species $i$ have biomass given by $b_{1 i}$ and allocation to root fraction given by $u_{1 i}$ with dynamics given by

$$
\frac{\mathrm{d} b_{1 i}}{\mathrm{~d} t} \frac{1}{b_{1 i}}=\frac{r N u_{1 i}}{N+k_{N}}-R-d
$$

and light-limited species $j$ have biomass given by $b_{2 j}$ and allocation to root fraction given by $u_{2 j}$ with dynamics given by

$$
\frac{\mathrm{d} b_{2 j}}{\mathrm{~d} t} \frac{1}{b_{2 j}}=\frac{r L\left(1-u_{2 j}\right)}{L+k_{L}}-R-d .
$$

The allocation fractions are required to satisfy the constraints

$$
\begin{aligned}
& 0 \leqslant u_{1 i} \leqslant u_{m} \\
& u_{m} \leqslant u_{2 j} \leqslant 1 .
\end{aligned}
$$

Under this model, each group will have a dynamic which conforms to eqn (1). Since $u_{m}$ is a function of $N$ and $L$ (both dynamic variables), it will, in general, change with time. Strictly speaking, in order for the solutions to (5) and (6) to match (1) it would be required that if $u_{m}$ changed in such a way to violate the inequalities (7) or (8), species must then change types at such a point. That is, a species must switch from a light-limited type to a nutrient-limited type or vice versa while maintaining the same strategy value. However, we do not have to deal with this difficulty since we use eqn (1) in our simulations (given later). Equations (5) and (6) will be used only to obtain the G-functions for use with the ESS maximum principle as given below.

Note that the effect of one species' strategy on another is not contained in the growth eqns (5) and (6). This effect is, in fact, cross-coupled only through the resource equations which are given in the next section. This results from modelling a community in which species interact only through the consumption of common resources. Since there are no evolutionary parameters associated with $N$ and $L$, the nutrient and light equations are not expressed in terms of
G-functions. For the modified model given above, two G-functions need to be defined: one for the nutrient-limited type and one for the light-limited type. Since the fitness function for a given species of either type contains only the strategy of that species, the G-functions for each type are given by replacing $u_{1 i}$ with $u$ and $u_{2 j}$ with $v$ in the r.h.s. of eqns (5) and (6). The nutrient-limited types, which we refer to as $N_{\text {plants }}$ have a G-function given by

$$
G_{1}=\frac{r N u}{N+k_{N}}-R-d
$$

and the light-limited types, $L_{\text {plants }}$, have a G-function given by

$$
G_{2}=\frac{r L(1-v)}{L+k_{L}}-R-d .
$$

While all $N_{\text {plants }}$ are evolutionarily identical and all $L_{\text {plants }}$ are evolutionarily identical, the $N_{\text {plants }}$ are not evolutionarily identical to the $L_{\text {plants. }}$. Although each type uses the same kind of strategy, root allocation fraction, each type is constrained to a different set of strategy choices defined by (7) and (8).

We assume that for fixed constants, there exists a

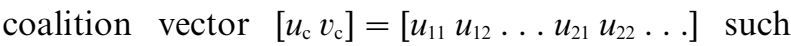
that there exists equilibrium solutions for the consumers, $b^{*}$, and the resources $N^{*}$ and $L^{*}$. The ESS maximum principle, for this situation, requires that if $\left[u_{\mathrm{c}} v_{\mathrm{c}}\right]$ is an ESS then the equilibrium solution to (9), $G_{1}^{*}$, must take on a maximum with respect to each component of $u_{\mathrm{c}}$ and the equilibrium solution to (10), $G_{2}^{*}$, must take on a maximum with respect to each component of $v_{c}$ (Vincent, 1995).

Note that

$$
G_{1}^{*}=\frac{r N^{*} u}{N^{*}+k_{N}}-R-d
$$

and

$$
G_{2}^{*}=\frac{r L^{*}(1-v)}{L^{*}+k_{L}}-R-d
$$

are independent of the number of species so that they are valid for all possible coalitions; in other words, any number of potentially coexisting species. Since $u$ and $v$ appear linearly in the $G^{*}$ functions and are bounded by $0 \leqslant u \leqslant u_{m}$ and $u_{m} \leqslant v \leqslant 1$, the only coalition vector which will satisfy the maximum principle is given by $\left(u_{11}=u_{m}^{*}, u_{21}=u_{m}^{*}\right)$ where $u_{m}^{*}$ is given by (4) evaluated at $L=L^{*}$ and $N=N^{*}$. Thus the ESS maximum principle is satisfied by a coalition of 2 , with an identical strategy $\left(u_{m}^{*}\right)$ for each resource-limited type. Since a species is identified by its strategy, we may interpret this result as either coexistence of one species of $N_{\text {plants }}$ with one species of 
$L_{\text {plants }}$ both using the same strategy (with the same fitness) or as one species, of either type, with the single ESS strategy $u_{m}^{*}$. The latter is more the biological intent of R \& P's model so we will call this an ESS coalition of one.

The ESS strategy is the same as the final dominant strategy obtained by R \& P. They tested the stability of this final dominant using an analysis of the Jacobian matrix. However, if an ESS exists, then by definition it is stable with respect to both variations in population density and variations in strategy and a separate stability test is not necessary (Vincent et al., 1995). Note that the ESS solution obtained here does not require specific equations for nutrient and sunlight, only that equilibrium values are available so that $u_{m}$ can be calculated.

\section{Simulation Results}

The strategies used by a given species do not change over an individual's lifetime. However, since strategies are adaptive parameters, they may change for the species over evolutionary time through natural selection. This process will produce a strategy dynamic (a change in strategy over evolutionary time) which drives a species toward the ESS. The ESS maximum principle allows us to predict the ultimate outcome of this evolutionary process (provided the environment is stable long enough for an equilibrium solution to be obtained). If we want to observe the evolutionary process (as opposed to just predicting its outcome) then a strategy dynamic must be added to the system model. Instead of seeding our simulation with a range of species, we use a strategy dynamic based on the G-function by assuming that every strategy $u$ has a small variation in strategies around it. This variation allows a natural selection process to take place (Vincent et al., 1993; Vincent, 1995). For small symmetric variation in a scalar strategy, $u_{i}$, the following strategy dynamic is obtained.

$$
\dot{u}_{i}=\sigma^{2}\left(\frac{\partial G_{i}}{\partial u_{i}}\right)
$$

where $\sigma$ is the variance in strategy about the mean $u_{i}$. This expression has much in common with the way heritability and genetic and phenotypic variances scale evolutionary rates in quantitative genetic models (Taper \& Case, 1985). It is also in agreement with Fisher's fundamental theorem of natural selection (Fisher, 1930) which states, "[t]he rate of increase in fitness of any organism at any time is equal to its genetic variance in fitness at that time". The gradient of the G-function represents the change in fitness for a given change in strategy. Hence the greater the genetic variance, the greater the change in fitness and by (13) the more rapidly an organism will evolve toward an ESS.

It is of interest to compare our simulation model, under evolution, to R \& P's simulation model of succession, which they use to test their analytical results. From R \& P the nutrient dynamics are given by

$$
\begin{aligned}
\frac{\mathrm{d} N}{\mathrm{~d} t}= & a\left(T-N-\sum_{i=1}^{n_{i}} p b_{1 i}+\sum_{j=1}^{n_{j}} p b_{2 j}\right) \\
& -\sum_{i=1}^{n_{i}} p b_{1 i}\left[G_{1}\left(u_{1 i}\right)+d\right] \\
& -\sum_{j=1}^{n_{j}} p b_{2 j}\left[G_{2}\left(u_{2 j}\right)+d\right]
\end{aligned}
$$

and the light availability is given by

$$
L=\frac{L_{0}}{1+\sum_{i=1}^{n_{i}} \alpha b_{1 i}\left(1-u_{1 i}\right)+\sum_{j=1}^{n_{j}} \alpha b_{2 j}\left(1-u_{2 j}\right)}
$$

where

$T=$ total soil nutrient in habitat

$a=$ mineralization rate

$p=$ plant tissue nutrient concentration

$L_{0}=$ solar constant

$\alpha=$ light decay rate per unit leaf biomass

and where $n_{i}+n_{j}=n$ (total number of species) with the understanding that if $n_{i}=0$ then the summation is zero and likewise for $n_{j}$. Note that $G_{1}$ is evaluated at $u_{1 i}$ and $G_{2}$ is evaluated at $u_{2 j}$. In what follows, we use the same constants given in R \& P: $a=0.3, r=5$, $k_{N}=k_{L}=1, p=0.1, R=0.5, \alpha=0.001, L_{0}=2$.

There are two ways to determine the ESS strategy: by direct calculation using the ESS maximum principle or through simulation using strategy dynamics. Since the ESS maximum principle predicts that the ESS strategy is given by $u_{m}^{*}$, we can determine $u_{m}^{*}$ by solving four equilibrium equations. They are (4), (15), the r.h.s. of (5) or (6) set equal to zero with $u_{1 i}$ and $u_{2 j}$ set equal to $u_{m}$ and $b_{1 i}=b_{2 j}=b$, the r.h.s. of (14) set equal to zero with $u_{1 i}$ or $u_{2 j}$ set equal to $u_{m}$ and $b_{1 i}=b, b_{2 j}=0$ or $b_{1 i}=0, b_{2 j}=b$, depending on whether (5) or (6) is used. Simultaneously solving these equations (using MATHEMATICA) with $T=5$ and $d=0.5$ we obtained: $u_{m}^{*}=0.700517$, along with $b^{*}=17.2515, N^{*}=0.399587, L^{*}=2.01039$.

This result may now be compared with a simulation which uses strategy dynamics. For simulation, we use 
the plant growth dynamics given by (1). In terms of the G-functions, this is equivalent to calculating $G_{1}$ and $G_{2}$ from (9) and (10) for a given value of $u$ and $v$ and then adding the requirement that

$$
\text { IF } G_{1}<G_{2} \text { THEN } G=G_{1} \text { ELSE } G=G_{2} .
$$

Since we are seeking an ESS coalition of one, it is sufficient to start with just one species. However, we obtain a more interesting simulation if we start with two species.

Using (13) for our strategy dynamic and choosing $\sigma^{2}=0.005, \quad b_{1}(0)=b_{2}(0)=0.5, \quad N(0)=10$, $u_{1}(0)=0.3, u_{2}(0)=0.6$, we obtained the results shown in Figs 1-5. Figure 1 shows the G-functions as a function of root allocation fraction at time $t=0$. As the system moved toward equilibrium, the intersection of the G-functions approached zero as illustrated in Fig. 2 (recall that at equilibrium $G^{*}=0$ at the ESS and that the ESS is $u_{m}^{*}$ ). We see from Fig. 3 that species 1 started as an $N_{\text {plant }}$ (nutrient-limited plant: $b_{1}$ starting with a strategy below the initial value of $u_{m}$ ) and that species 2 started as an $L_{\text {plant }}$ (light-limited plant: $b_{2}$ starting with a strategy above the initial value of $u_{m}$ ). Both evolved toward $u_{m}^{*}$ but the $L_{\text {plants }}$ reached $u_{m}^{*}$ first. Figure 4 illustrates the nutrient and light dynamics for this simulation. Nutrient levels dropped rapidly while light levels remained nearly constant. Since nutrients became more limiting than light, it makes sense that the $L_{\text {plants }}$ reached $u_{m}^{*}$ first. Since both plant types evolved to the same strategy, they may be considered the same species. As illustrated in Fig. 5, both plants were able to obtain

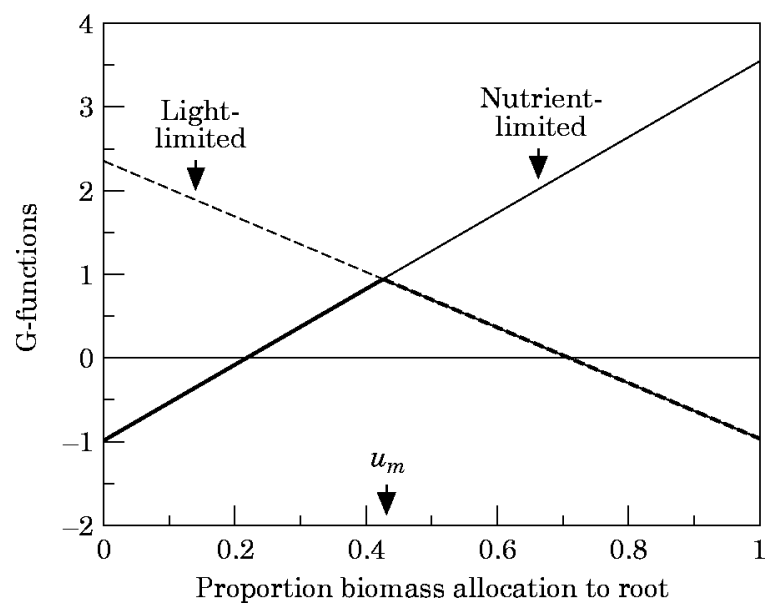

FIG. 1. The solid line depicts the nutrient-limited type of eqn (9) and the dotted line depicts the light-limited type of eqn (10). The graph depicts the G-functions for initial conditions given in the text. The intersection of the two curves is $u_{m}$ and is given in eqn (4). Since eqn (1) was used for simulation, to satisfy the MIN function, only the lower portions of each line (shown in bold) were used.

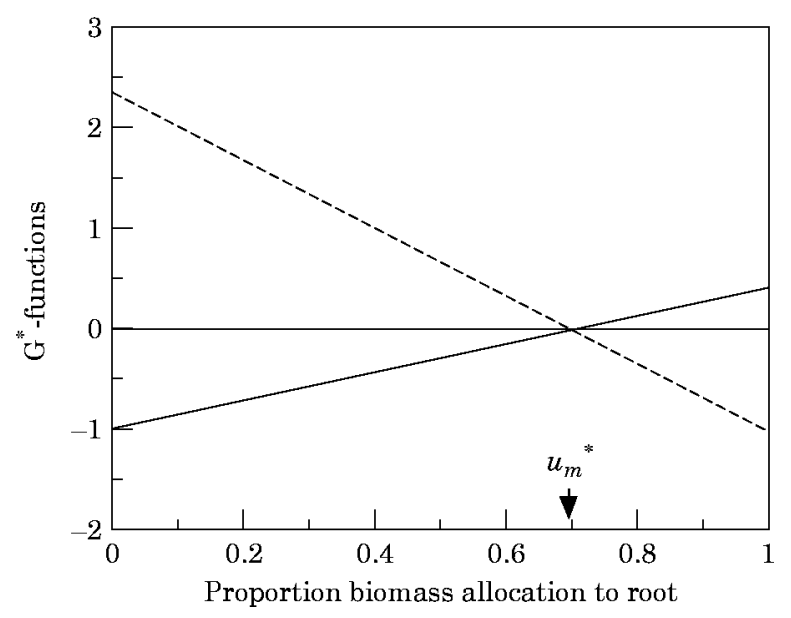

FIG. 2. The solid line depicts eqn (11) and the dotted line depicts eqn (12) for the G-functions as a function of root allocation strategy at equilibrium. During the course of simulated evolution, the intersection of these two lines moved from its initial position in Fig. 1 to the value of $G^{*}=0$ at equilibrium where $u_{m}=u_{m}^{*}$.

the ESS strategy with a combined population number $b_{1}^{*}+b_{2}^{*}=17.247$ (compare with previous MATHEMATICA result $b=17.252$ ). Additional final equilibrium values obtained in the simulation were $u_{m}^{*}=0.699, N^{*}=0.401, L^{*}=1.990$. These numbers are in good agreement with those predicted by direct application of the ESS maximum principle. However, we could have started with just one plant and obtained the same result.

\section{Arrested Evolution?}

In our simulation, evolution drives the system rather than succession (as in R \& P; Tilman, 1988).

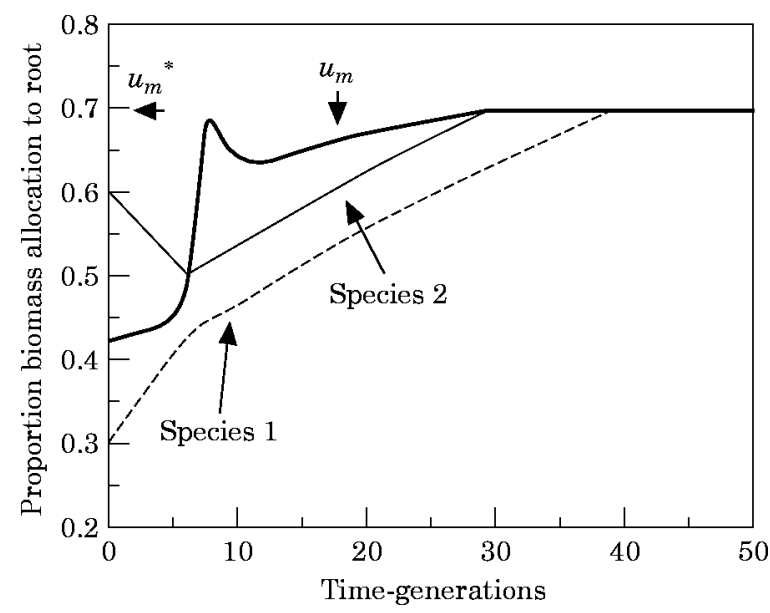

FIG. 3. $u_{m}$, the optimal strategy for a given $N$ and $L$ (R \& P), changed over time. This is shown in bold. The evolution, over time, of the initial strategies $u_{1}=0.3$ (species 1 ) and $u_{2}=0.6$ (species 2) are shown as dotted and solid lines, respectively. Note that both strategies evolved to the same solution (they became identical species) but that species 2 reached the equilibrium solution first. 


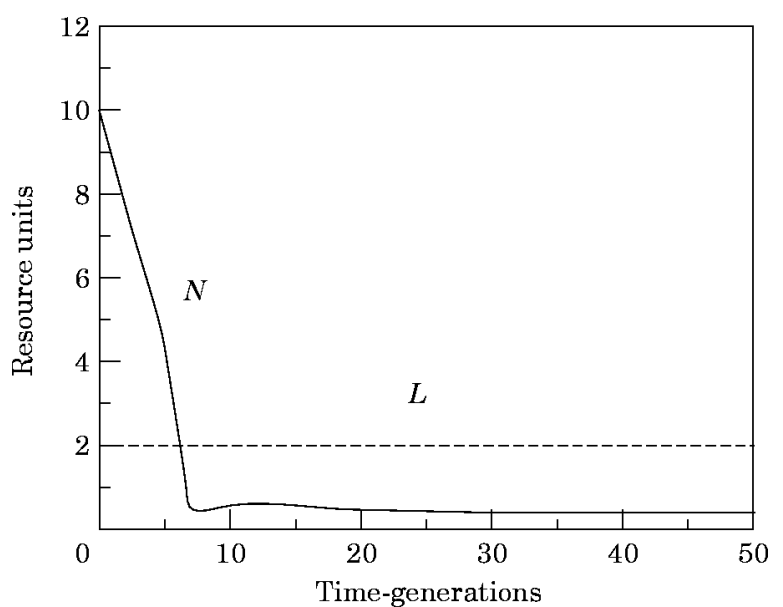

FIG. 4. The change in nutrients (solid line) and the change in light (dotted line) over time are taken from eqns (14) and (15), respectively.

Instead of seeding the system with all possible strategies (as done by $\mathrm{R} \& \mathrm{P}$ ), we can start with any two strategies and allow them to evolve to the evolutionarily stable strategy. The ESS, as given by $u_{m}^{*}$, is the same as the final dominant strategy under equilibrium conditions calculated by R \& P. (Note that the final dominant strategy calculated by $\mathrm{R} \& \mathrm{P}$ had a value of 0.67 . The difference between this value and our calculated value of 0.70 is, no doubt, due to the value of $d$ used, which was unspecified in $\mathrm{R} \& \mathrm{P}$ except for a range of values. In our model, $d=0.5$.) At conditions other than equilibrium, $u_{m}$, as given by (4), defines the optimal strategy for the current resource conditions. Thus, as our system evolves, the initial strategies must "chase a moving target" as $u_{m}$ changes. The analog for succession, as found by

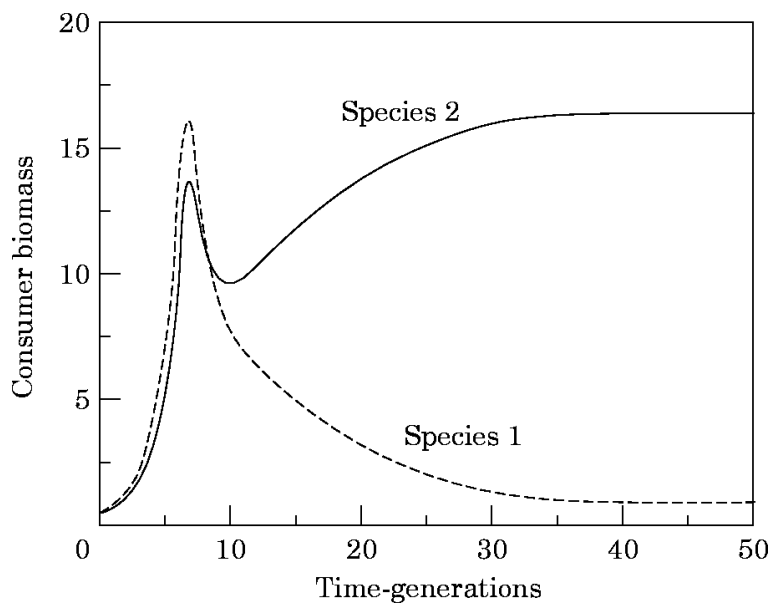

FIG. 5. Populations of species 1 (dotted) and species 2 (solid) are shown vs. time. Since both species 1 and species 2 evolved to the same strategy, their populations can be summed to give the equilibrium population of the ESS strategy, $u_{m}^{*}$.
$\mathrm{R} \& \mathrm{P}$, is that the initial dominant strategy changes the initial resource conditions of the community which, in turn, changes the optimal strategy. The seeded strategy that is closest to the new optimal strategy becomes dominant.

When $\mathrm{R} \& \mathrm{P}$ ran simulations of their model, under certain conditions they found "arrested successions". That is, succession from the initial dominant strategy to the final dominant strategy $u_{m}^{*}$ did not occur. Arrested successions occurred when a range of allocation strategies between the initial and final dominant strategy values was eliminated. Instead, succession proceeded from the initial dominant value to a strategy that was closest to the final dominant value. For example, by removing the set of strategies $0.46-0.60$ from the pool of strategies ( $\mathrm{R} \& \mathrm{P}$ used seeded strategies ranging from 0.01 to 0.99 at 0.01 unit intervals) and setting the direction of selection, determined by the terms $T$ and $d$, such that nutrient specialists were favored over light specialists (see $\mathrm{R} \& \mathrm{P}$ for details), succession proceeded from the initial dominant 0.40 to the strategy 0.45 . Although the final dominant value was fed into the simulation, it could not displace 0.45 .

Can we get arrested evolution in a similar fashion? To simulate arrested evolution, we eliminated a range of $u(0.45<u<0.61)$ so that $u$ could not evolve to any of the eliminated strategies. Like R \& P, we found that a strategy starting at 0.45 could dominate a strategy starting at $u_{m}^{*}=0.70$ provided that evolution was not allowed to occur $(\sigma=0)$. In general, neither strategy will coincide with $u_{m}$ during the simulation. While this system reached local stability, it was not evolutionarily stable since the intersection of the G-functions never reached zero. When evolution was allowed to occur $(\sigma \neq 0)$, the species using the initial strategy 0.45 grew rapidly to a large biomass initially, but as time passed, the species using the initial strategy 0.70 evolved to $u_{m}$ and tracked $u_{m}$ until the equilibrium value of 0.70 was again obtained. This strategy became dominant since at equilibrium $u_{m}^{*}$ is an ESS strategy. Thus, as long as species can evolve, there will be no "arrested evolution". This result assumes that the system will be provided with strategies above 0.65 . In summary, "arrested evolution" analogous to "arrested succession" can occur in our simulations (even when the ESS strategy is present) only if there is no evolution.

\section{Coexistence}

When a G-function is linear with respect to the strategy $u$, then a single ESS strategy (coalition of 


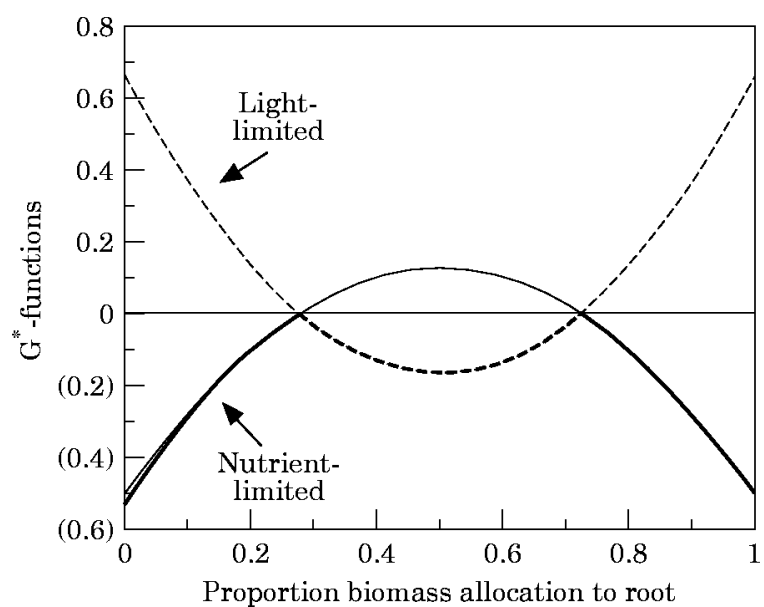

FIG. 6. The solid line depicts eqn (17) and the dotted line depicts eqn (18) for the G-functions as a function of root allocation strategy at equilibrium. The choice of function used in the simulation was determined by the MIN value of both eqns. Analogous to eqn (1) in Fig. 1, only the portions of each line that are shown in bold were used in the simulation.

one) must occur at the upper boundary set by the constraints on $u$ provided that the slope of the G-function is non-zero. This follows geometrically from the fact that a non-zero linear function can have no maxima or minima except at the function's boundaries. [If a linear function is flat (i.e. equal to zero), then every point along that line has the same minima.] The model presented here contains two linear G-functions. We see from Figs 1 and 2 that the nutrient-limited growth curve takes on a maximum when $u=1$. That is, if nutrients are limiting, then maximal growth rate can be obtained for the biologically unrealistic case of total allocation of photosynthate to roots. Likewise, the light-limited growth curve takes on a maximum when there is total allocation to leaves. However, with constraints (7) and (8), the ESS strategy must occur at $u_{m}^{*}$, the upper boundary of both curves set by the constraints (7) and (8) at equilibrium.

For coexistence to occur between two or more species, the G-function must take on a maximum at two or more strategies (Vincent et al., 1993). One way to obtain this solution is for the G-function to be linear but with zero slope. In this case, any number of strategies may coexist since any particular strategy may be invaded, but not displaced, by another (Vincent et al., 1993). The G-function for this model can only take on a zero slope for unrealistic conditions where $r, N$ or $L=0$ and the trivial condition where only two strategies, all root and all shoot, exist. Thus, in order to obtain coexistence between two or more strategies, a nonlinear dependance of the G-function on $u$ must be introduced. Figure 6 is an example of a G-function with an ESS coalition of two.

The following nonlinear $\mathrm{G}^{*}$-functions were used to generate Fig. 6:

$$
\begin{gathered}
G_{1}^{*}=\frac{r N^{*}\left(u-u^{2}+0.2\right)}{N^{*}+k_{N}}-R-d \\
G_{2}^{*}=\frac{r L^{*}\left(-1+v+(1-v)^{2}+0.5\right)}{L^{*}+k_{L}}-R-d
\end{gathered}
$$

where $u$ is the dummy variable for $u_{1 i}$ and $v$ is the dummy variable for $u_{2 j}$. The form of these $\mathrm{G}^{*}$-functions is analogous to (1). Although these functions were generated to fit the type of curves found in Fig. 6, eqn (17) might be interpreted biologically to mean that growth rate is greatest at intermediate allocation to roots while eqn (18) might be interpreted biologically to mean that growth rate is greatest at either very high or very low allocation to shoots. Figures 7-8 show a simulation of evolution to an ESS coalition of two allocation strategies and the growth of these populations over time, respectively.

The G-function approach allows us to rapidly determine and generate necessary conditions for coexistence between two or more species. This approach is different from that employed by Tilman (1980) who derived necessary conditions for coexistence based on a Jacobian matrix analysis of his combined set of consumer-resource equations. We note that this type of analysis depends on the forms of both the consumer and resource equations and

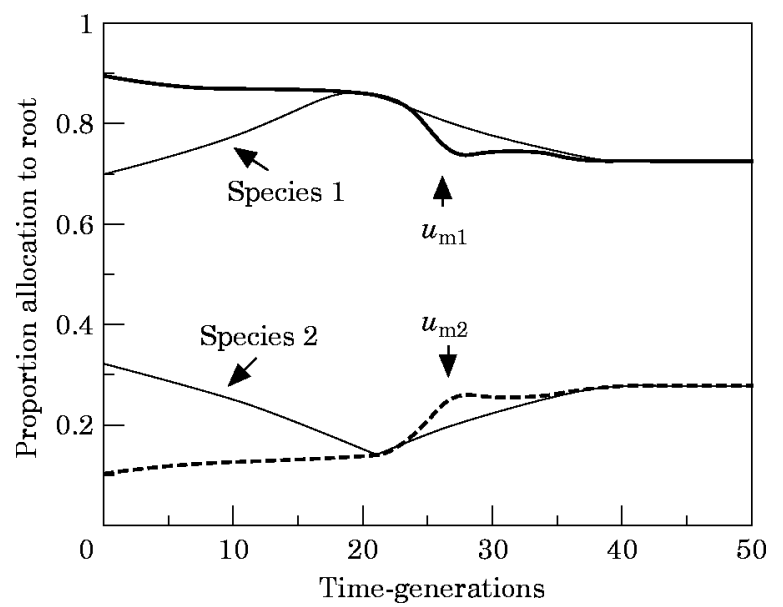

FIG. 7. Since the G-functions given in eqns (17) and (18) intersected at two points with respect to allocation strategies, this gives two $u_{m}$. Both of these intersection points changed with time until the equilibrium solutions were reached (solid and dotted bold lines). Initial strategies $u_{1}=0.7$ and $u_{2}=0.32$ for species 1 and 2 , respectively, evolved to two different strategies and are shown as solid and dotted lines, respectively. 


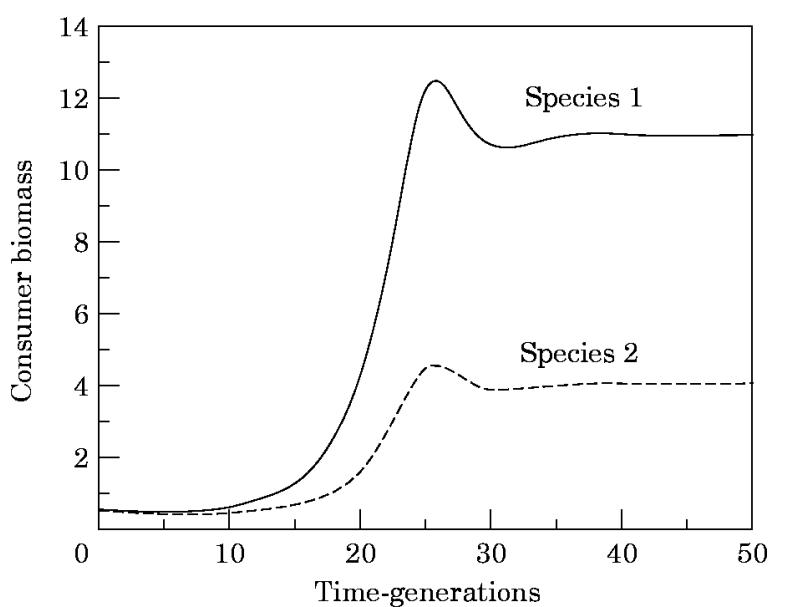

FIG. 8. Populations of species 1 (solid) and species 2 (dotted) are shown over time.

thus general conclusions about necessary conditions for coexistence may not apply when the forms of resource or consumer equations are changed.

\section{Implications for Coexistence}

From our ESS approach, we confirmed what Tilman (1988) showed via simulation and R \& P proved via Jacobian matrix - that for this type of consumer-resource root-shoot allocation model, only one species can exist at equilibrium. Although the solution is the same, the methods for obtaining conditions for coexistence (and the reason why only one species can exist) differed. R \& P used the conditions for coexistence derived by Tilman (1980) and discussed in Tilman (1982): that a two-species equilibrium point is stable if each species consumes relatively more of the resource that most limits its own growth. Tilman (1980) derived these conditions using general equations for both the dynamics of the consumers and the resources. Although the light resource equation used by $\mathrm{R} \& \mathrm{P}$ was not of the same form as given in Tilman's (1980) derivation (R \& P's equation equilibrated instantaneously), their conclusion that the two-species equilibrium point is unstable because leafier species consume more light (the resource that less limits them) than rootier species appears valid. As R \& P state, this is because light is consumed in direct proportion to biomass while nutrients are consumed in proportion to net carbon gain. However, the conditions for coexistence derived using the ESS approach do not depend on the form of the nutrient dynamic equations (and hence the consumption vectors). Instead, the ESS approach shows us that the number of coexisting species depends on the number of maxima in the $G^{*}$ equation. That is, conditions for coexistence depend on the consumer growth equation, not consumption in the resource equations. It is easy to tell from the graph of $G^{*}$ vs. root allocation strategy, $u$, that only one maxima exists for the growth equation described by $\mathrm{R} \& \mathrm{P}$. It is also easy to show that coexistence of two or more species is possible only if the G-functions are made nonlinear with respect to $u$. Although it is not examined in this paper, it may be enlightening to determine whether the stability rules based on consumption of resources that Tilman (1980) derived are directly connected to ESS maxima via a mathematical relationship.

How realistic are nonlinear G-functions? Nonlinear relationships, as given by (17) and (18), between biomass allocation and growth could occur if the efficiency in converting or capturing a resource changed with biomass allocation or if loss rates (e.g. respiration, disturbance, herbivory, etc.) changed with biomass allocation. For example, the humpshaped curve shown in Fig. 6 could model a peak in efficiency at intermediate biomass allocation to roots while the u-shaped curve shown in Fig. 6 might model a high loss rate at intermediate biomass allocation to shoots. The former scenario might be a case where the efficiency of nutrient uptake scales with root mass at lower root allocation but at higher root allocation, most of the root biomass becomes more efficient at nutrient storage instead of uptake. The latter scenario might be a case where loss to herbivory is low at low leaf biomass because herbivores switch to more common species and loss is low at high leaf biomass because herbivores are food saturated but loss is high otherwise.

Note that higher order nonlinear relationships could increase the number of intersecting peaks between the two G-functions, thus allowing more than two species to coexist at equilibrium - even on just two limiting resources. Thus, while the root-shoot allocation model of $\mathrm{R} \& \mathrm{P}$ results in a monoculture at equilibrium, by allowing nonlinear complexities (caused by interactions between biomass allocation and other parameters), coexistence between species with different allocation strategies becomes possible.

We would like to thank all of the people that reviewed this paper. Their comments were appreciated very much.

\section{REFERENCES}

Bloom, A. J., Chapin, F. S. III \& Mooney, H. A. (1985). Resource limitation in plants - an economic analogy. Ann. Rev. Ecol. Syst. 16, 363-392.

Brown, J. S. \& Vincent, T. L. (1986). Predator-prey coevolution as 
an evolutionary game. In: Applications of Control Theory in Ecology (Y. Cohen, ed.). Berlin: Springer-Verlag.

BRown, J. S. \& VINCENT, T. L. (1987). A theory for the evolutionary game. Theor. Popul. Biol. 31, 140-166.

Brown, J. S. \& Vincent, T. L. (1992). Organization of predator-prey communities as an evolutionary game. Evolution, 46, 1269-1283.

Chapin, F. S. III (1980). The mineral nutrition of wild plants. Ann. Rev. Ecol. Syst. 11, 233-260.

Fisher, R.A. (1930). The Genetical Theory of Natural Selection. Oxford: Clarendon Press.

GRIME, J. P. (1977). Evidence for the existence of three primary strategies in plants and its relevance to ecological and evolutionary theory. Am. Nat. 111, 1169-1194.

Maynard Smith, J. (1982). Evolution and the Theory of Games. Cambridge: Cambridge University Press.

Reynolds, H. L. \& Pacala, S. W. (1993). An analytical treatment of root-to-shoot ratio and plant competition for soil nutrient and light. Am. Nat. 141, 51-70.

TAPER, M. L. \& CASE, T. J. (1985). Quantitative genetic models for the coevolution of character displacement, Ecology, 66, $355-371$.
Tilman, D. (1980). Resources: a graphical-mechanistic approach to competition and predation. Am. Nat. 116, 362-393.

Tilman, D. (1982). Resource Competition and Community Structure. Monographs in Population Biology 17. Princeton: Princeton University Press.

Tilman, D. (1988). Plant Strategies and the Dynamics and Structure of Plant Communities. Monographs in Population Biology 26. Princeton: Princeton University Press.

VINCENT, T. L. (1995). The ESS maximum principle as a tool for modeling and managing biological systems. In: Control and Game Theoretic Models of the Environment (Filar, J. A. \& Carraro, C., eds), pp. 259-278. New York: Birkhäuser Boston. VINCENT, T. L. \& BROWN, J. S. (1988). The evolution of ESS theory. Ann. Rev. Ecol. Syst. 19, 423-443.

Vincent, T. L. \& BRown, J. S. (1989). The evolutionary response of systems to a changing environment. Appl. Math. Comp. 32, 185-206.

Vincent, T. L., Cohen, Y. \& Brown, J. S. (1993). Evolution via strategy dynamics. Theor. Popul. Biol. 44, 149-176.

VINCENT, T. L., VAN, M. V. \& GoH, B. S. (1995). Ecological stability, evolutionary stability, and the ESS maximum principle. Evol. Ecol. In Press. 